\title{
Anterior cingulate cortex in schema assimilation and expression
}

\author{
Szu-Han Wang, ${ }^{1}$ Dorothy Tse, and Richard G.M. Morris \\ Centre for Cognitive and Neural Systems, The University of Edinburgh, EH8 9JZ Edinburgh, United Kingdom
}

\begin{abstract}
In humans and in animals, mental schemas can store information within an associative framework that enables rapid and efficient assimilation of new information. Using a hippocampal-dependent paired-associate task, we now report that the anterior cingulate cortex is part of a neocortical network of schema storage with NMDA receptor-mediated transmission critical for information updating, and AMPA receptor-mediated transmission required for the expression and updating of stored information.
\end{abstract}

[Supplemental material is available for this article.]

Humans form coherent frameworks of knowledge called "mental schemas" (Bransford 1979; van Kesteren et al. 2010). Schemas provide a framework for organizing information, for its recall and the efficient incorporation of new information. Schemas are not restricted to humans. It has been recently reported that rodents can concurrently learn multiple flavor-place paired associations (PAs) and form schemas that allow for rapid future learning (Tse et al. 2007). The discovery of schemas in rodents has implications for existing theories of cortical memory consolidation (McClelland et al. 1995; Squire and Alvarez 1995; Nadel and Moscovitch 1997), but their anatomical basis is unclear. In this study, building on work comparing recent vs. remote memory with respect to consolidation (Frankland and Bontempi 2005), we examined the role of the anterior cingulate cortex (ACC) in the expression of stored PAs within a schema and the assimilation of new information.

In rats, PA learning that underlies schema formation is hippocampal-dependent (Tse et al. 2007). However, cortical structures are likely involved in the parallel encoding of new information and schema creation (Tse et al. 2011). The consolidation of new information into schemas engages a rapid (within $48 \mathrm{~h}$ ) consolidation process (Tse et al. 2007) such that the integrity of the hippocampus is, after this time, no longer necessary for memory retrieval. These observations prompt the question: What neocortical structures are essential for rapid assimilation into and information retrieval from schema? Because systems memory consolidation studied in experimentally naive animals has previously been shown to take several weeks, it has hitherto been thought that there is a gradual time-dependent process of brain "reorganization" that occurs after learning (Frankland and Bontempi 2005), such that brain areas involved in retrieving recently acquired memory (e.g., hippocampus) (Wang et al. 2009) differ from those mediating remote memory (e.g., ACC) (Frankland and Bontempi 2005; Teixeira et al. 2006). The concept of schemas challenges the necessity of gradual reorganization after learning as it raises the possibility that memory trace networks can be gradually created, but then rapidly updated in association with the activation of plasticity-associated genes in hippocampus and neocortex. It was therefore of interest to ask whether ACC was part of the cortical network supporting schemas.

\section{'Corresponding author}

E-mail s.wang@ed.ac.uk

Article is online at http://www.learnmem.org/cgi/doi/10.1101/Im.026336.112.
Rats were trained, one trial/day per flavor/location pair, on six separate PAs in an event arena (Fig. 1A; Supplemental Fig. S1) as described previously (Tse et al. 2007). Animals were cued with a particular flavor in the start box and trained to find more of that same flavor at its associated location in the arena. This training of PAs was repeated for the six trials of each day over 15 sessions in a randomized and counterbalanced manner. Over the course of training, the animals showed a significant improvement of performance (Fig. 1B; Supplemental Fig. S2A). They then received bilateral guide cannula implantation targeting the ACC (Supplemental Fig. S3) and, after recovery from surgery, were retrained with the six original PAs prior to pharmacological interventions, via infusion cannula, of new encoding and retrieval interspersed with further training sessions (inset in Fig. 1B; Supplemental Fig. S2B).

To examine whether the encoding of new PAs that could be successfully assimilated into the schema required neural transmission in the ACC, two new PAs were preceded by intra-ACC infusions of vehicle (saline) or 6-cyano-7-nitroquinoxaline-2,3-dione (CNQX), a competitive $\alpha$-Amino-3-hydroxy-5-Methyl-4- isoxazolePropionic Acid (AMPA)/kainate receptor antagonist (Fig. 1C). While performance during new PA acquisition was indifferent between drug conditions (Supplemental Fig. S4A,B), the longterm memory of the new information during a nonrewarded probe test conducted $24 \mathrm{~h}$ later was impaired by CNQX (Fig. 1C). Similar results were secured following intra-ACC infusions of $\mathrm{N}$-Methyl-D-aspartic acid (NMDA) receptor blocker, D-(-)-2Amino-5-Phosphonopentanoic acid (d-AP5) prior to encoding (Fig. 1C; Supplemental Fig. S4C,D), raising the possibility that NMDA receptor-dependent transmission, likely plasticity, is necessary for schema assimilation.

We then examined the retrieval of newly encoded PAs. We continued training the same animals using the original set of PAs over several sessions, followed by a single session of two trials with two new flavor-location PAs. Memory for these was examined the next day, the recall trials being preceded by CNQX, D-AP5, or vehicle infusions (Fig. 1D). The results showed with a different pattern that retrieving the long-term memory of new information was impaired by intra-ACC CNQX but not D-AP5. To examine whether synaptic transmission in ACC is also necessary for retrieving the original schema, we infused CNQX, D-AP5, or vehicle in the ACC before memory probe tests of the original schema PAs (Fig. 1E). Retrieval of PAs within the original schema was dependent on glutamatergic transmission in ACC, 
A

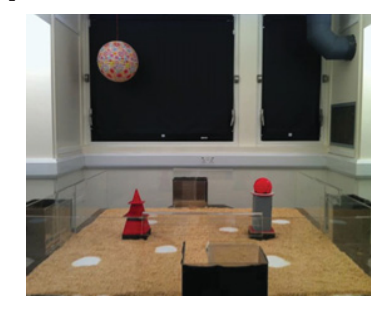

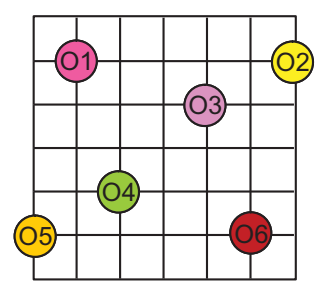

B

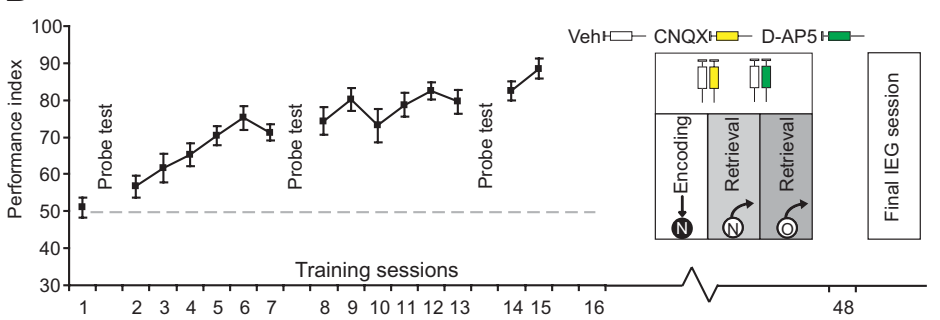

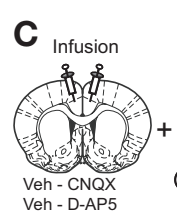

Veh - CNQX
Veh - D-AP5
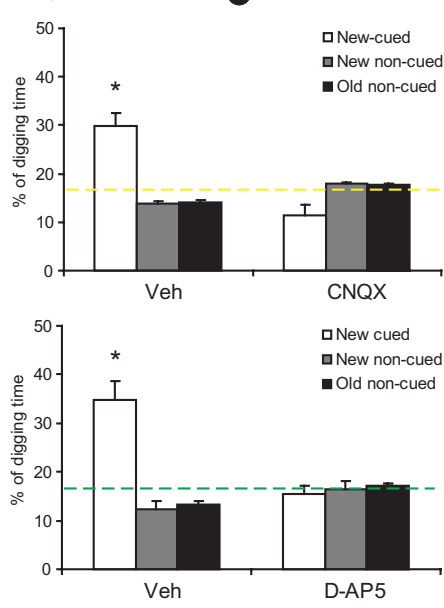

D
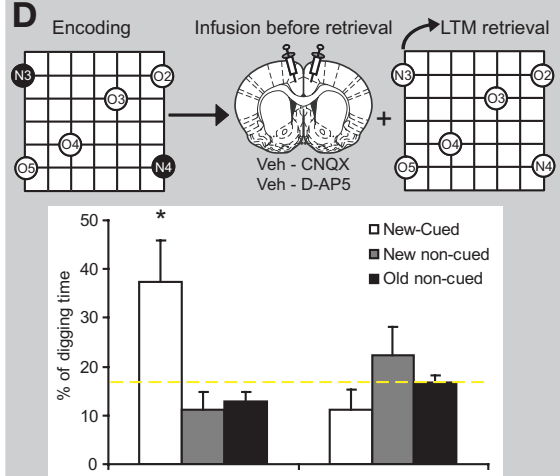

Veh

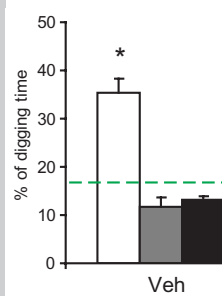

E
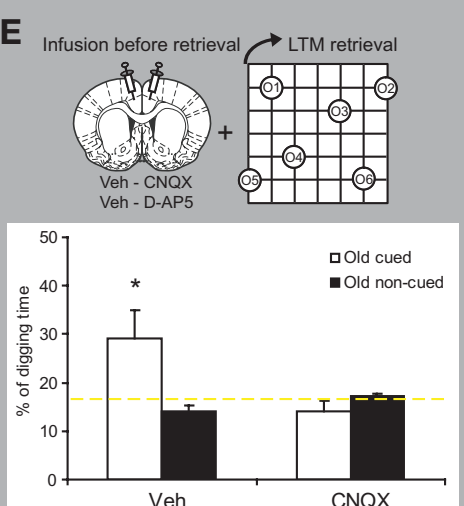

Veh

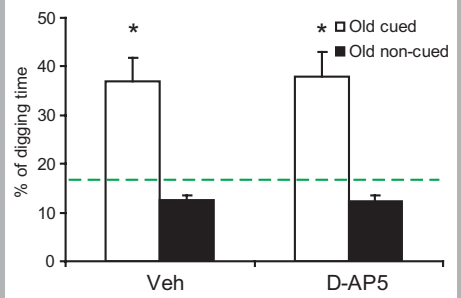

Figure 1. Glutamatergic mechanisms in ACC after schema learning. $(A)$ The event arena (left) and a schematic drawing of the six PAs (right). ( $B$ ) Performance gradually improved over training-increase of percentage of correct choice (linear trend $\left.F_{(1,15)}=210.02, P<0.001\right)$. $(C-E)(T o p)$ Procedures for drug infusions. (Bottom) Probe test results. (C) CNQX, D-AP5, or vehicle was infused 20 min before training of two new PAs (N1, N2, filled circles indicate rewarded encoding). In the probe test the next day, the animals were cued one of the new flavors at the start box (N1, N2, and two to five original locations; open circles to indicate nonrewarded testing). Digging in correct, new cued location was significantly better than chance $\left(t_{(13)}=5.03, P<0.001\right)$ after vehicle infusions but impaired after intra-ACC CNQX infusions (correct digging was lower than chance, $\left.t_{(13)}=-2.55, P<0.05\right)$. The interaction of treatment (Veh, CNQX) and digging type (at new cued, new noncued, old/original noncued wells) was significant $\left(F_{(2,26)}=34.8, P<0.001\right)$. Performance was impaired by intra-ACC D-AP5 infusions (correct digging was indifferent from chance, $t_{(13)}=-0.73$, $P=0.48)$ but, again, significantly better than chance in vehicle condition $\left(t_{(13)}=4.93, P<0.001\right)$. The interaction of treatment $($ Veh, D-AP5) and digging type was significant $\left(F_{(2,26)}=15.16, P<0.001\right)$. (D) Animals were trained on two new PAs (N3, N4). One day later, they received CNQX, D-AP5, or vehicle infusions, followed 20 min later by a probe test with the new flavor cues in the start box. Digging in the correct new cued location was significantly better than chance $\left(t_{(15)}=2.4, P<0.05\right)$ after vehicle infusions but impaired after CNQX infusions (correct digging indifferent from chance, $\left.t_{(15)}=-1.36, P=0.2\right)$. The interaction of treatment (Veh, CNQX) and digging type was significant $\left(F_{(2,30)}=8.3, P<0.01\right)$. Performance was significantly better than chance $\left(t_{(11)}=6.47, P<0.001\right)$ after both vehicle and D-AP5 infusions (correct digging was better than chance, $t_{(11)}=$ $5.39, P<0.001)$. The interaction of treatment (Veh, D-AP5) and digging type was nonsignificant $\left(F_{(2,22)}=2.4, P=0.11\right)$. $(E)$ Animals received CNQX, D-AP5, or vehicle infusions, followed 20 min later by nonrewarded testing with old/original flavor cues in the start box. Digging in the cued location was again significantly better than chance $\left(t_{(14)}=2.15, P<0.05\right)$ after vehicle infusions but impaired by $C N Q X$ (correct digging was indifferent from chance, $\left.t_{(14)}=-1.2, P=0.25\right)$. The interaction of treatment (Veh, CNQX) and digging type (at cued and noncued wells) was significant $\left(F_{(1,14)}=\right.$ 5.86, $P<0.05)$. Performance was significantly better than chance $\left(t_{(13)}=4.34, P<0.001\right)$ in both vehicle and intra-ACC D-AP5 infusion conditions (correct digging was better than chance, $t_{(13)}=4.17, P<0.01$ ). The interaction of treatment (Veh, D-AP5) and digging type was nonsignificant $\left(F_{(1,13)}=0.03, P=0.87\right)$. Means \pm 1 SEM. Dashed lines, chance level.

but not NMDA receptor-dependent mechanisms (including synaptic plasticity).

Two additional observations were made to rule out the possibility of nonspecific side effects of CNQX microinfusions into ACC. First, it was observed that both the performance index and the latency to retrieve all three pellets during encoding were comparable in CNQX and vehicle conditions (Supplemental Fig. S4A,B). This suggests an intact sensorimotor ability and motivation after CNQX infusion in the ACC. Similar performance under intra-ACC D-AP5 was also observed (Supplemental Fig. S4C,D). Second, infusion of CNQX alone into ACC without either training or testing had no effect on memory retrieval of the original flavorlocations on the next day (Supplemental Fig. S5A). Additionally, we observed that after CNQX infusion into ACC at the time of retraining of two original PAs, memory test at $3 \mathrm{~h}$ was intact (Supplemental Fig. S5B). This finding suggests that read-out from the hippocampus was intact and sufficient at this time and also implies that searching/procedural skills at a later time point were unaffected by CNQX infusions into ACC.

After all behavioral observations were complete, the expression of the immediate early gene zinc finger protein (Zif268; aka early growth factor1, egr1) in both ACC and hippocampus was 
A All animals were well trained
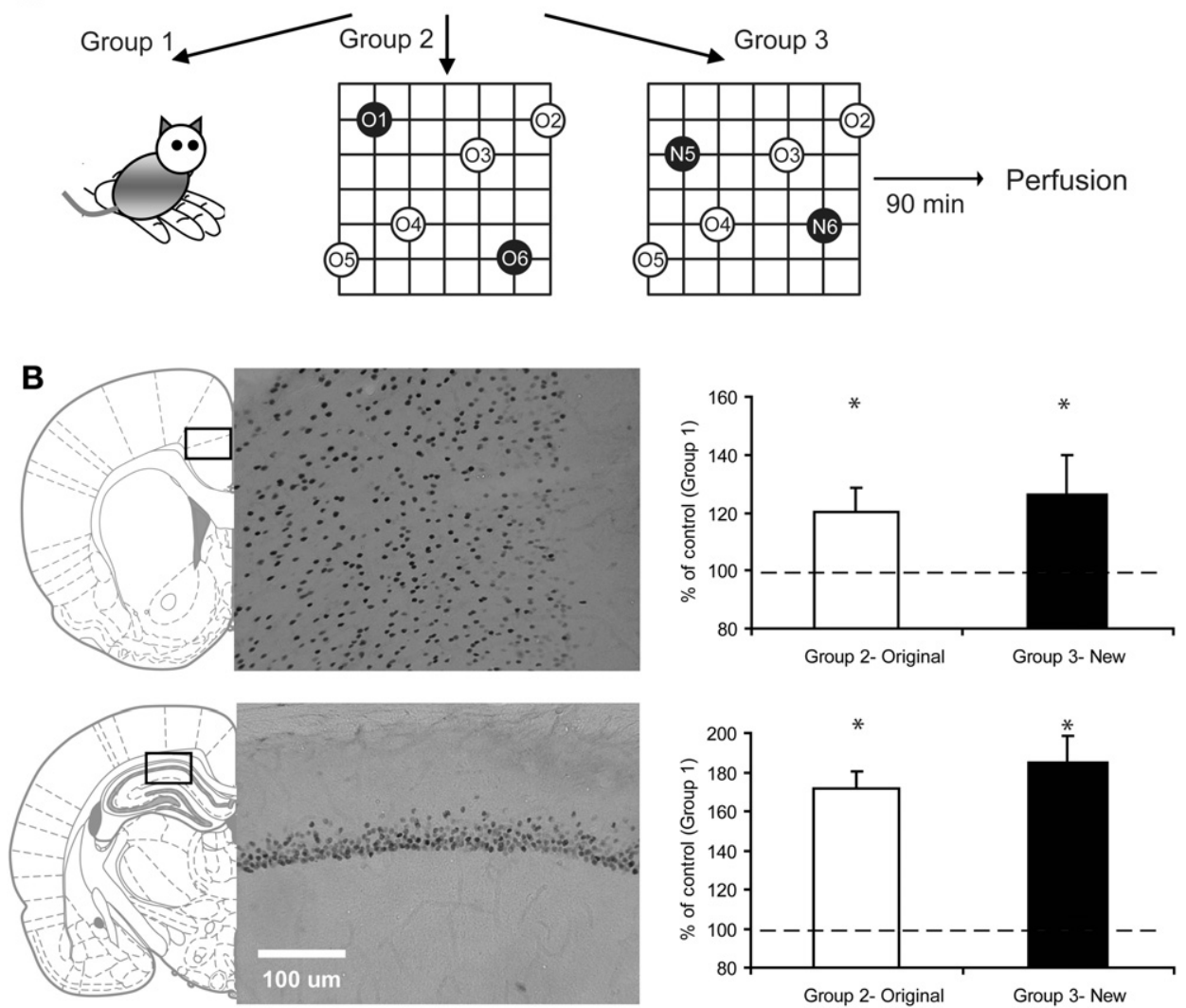

C
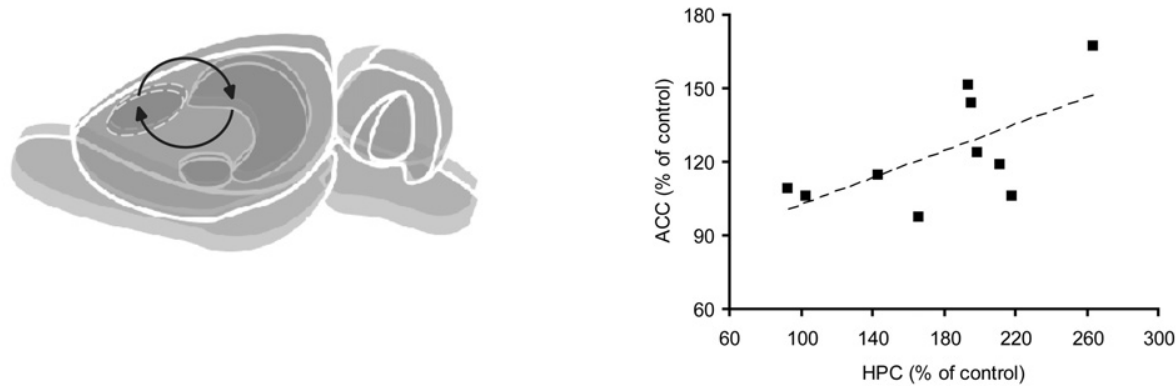

Figure 2. Correlated activities in ACC and HPC after animals were well-trained in the schema task. $(A)$ Experimental designs. Well-trained triads of rats were randomly assigned to Group 1: handled-only control, Group 2: retrained with two original PAs, Group 3: trained with two new PAs, followed by perfusions 90 min later. The brains were then prepared for neuroimaging by Zif268 immunohistochemistry and analyzed using regions of interest. (B) Representative pictures of Zif268+ cells in the ACC (bregma approximately -1 to $2 \mathrm{~mm}$, on top of corpus callosum connecting two hemispheres, an area of interest was selected at the cross-opposite zone from the top arch of corpus callosum) and HPC (post-bregma $\sim 2.7-4.3 \mathrm{~mm}$, an area of interest was selected at the center of dorsal CA1, right below the apex of the arch of corpus callosum). Groups 2 and 3 showed significantly more Zif268+ cells than Group 1 in both ACC (Group 2: $t_{(4)}=4.58, P<0.05$; Group 3: $\left.t_{(4)}=2.47, P=0.05\right)$ and HPC (Group 2: $t_{(4)}=3.9, P<0.05 ;$ Group 3: $t_{(4)}=3.13$, $P<0.05)$. (C) A significant positive correlation was observed between the number of Zif268+ cells in ACC and HPC $(r=0.63, P=0.05)$.

examined (Fig. 2A). Zif268 was chosen by virtue of its sensitivity in responding to neural activities associated with systems consolidation (Maviel et al. 2004). We observed a significant increase of Zif268+ cells in both ACC and HPC when the rats expressed their knowledge of a learned schema (original PAs) and when assimilating two new PAs (new PAs) relative to a subgroup of well-trained but handled-only control animals (Fig. 2B). We further examined Zif268 expression in the cortices adjacent to ACC, including the supplementary motor cortex (M2) and primary motor cortex (M1). The results showed that in M2, right next to ACC, there was a slight but nonsignificant increase of Zif268 expression (increase in original PAs group: $8 \% \pm 6.3 \%$, in new PAs group:
$14.6 \% \pm 11.3 \%$; both $P>0.3$ when compared with the control group). In M1, next to M2 and further away from ACC, there were no differences in Zif268 expression among these three groups (increase in original PAs group: $2.5 \% \pm 15.3 \%$, in new PAs group: $5.1 \% \pm 18.8$; both $P>0.7$ when compared with the control group).

After observing the significant increase of Zif268+ cells in both ACC and HPC, we further investigated whether Zif268 expression in these two areas is increased in a correlated fashion in the two PAs groups. We found that the magnitude of Zif268 increase in ACC was positively correlated with the magnitude of Zif268 increase in HPC $(r=0.63, P=0.05)$. There was no 
significant correlation between HPC and M1 or HPC and M2 (both $r \mathrm{~s} \leq 0.38, P>0.27)$. Together, these suggest a potential online functional interaction between ACC and HPC (Fig. 2C) during retrieving and/or updating information in the schema.

Our findings complement and extend our recent observation of a network of medial neocortical structures (prelimbic and retrospenial cortex) that are activated during updating (Tse et al. 2011). Here we show that neural transmission in ACC is also required for expressing and retrieving information from a cortical schema as well as for updating. NMDA receptor-dependent mechanisms, possibly plasticity, are required additionally for the updating associated with assimilation. While ACC was traditionally thought to be crucial for fundamental physiology such as pain perception, it was shown later on to play a role in pain-related memory processing (Tang et al. 2005) and decision making (Rushworth and Behrens 2008). For example, normal macaques can use the reinforcement history flexibly to guide their behavior when the response and outcome combination changes across sessions. Macaques with ACC sulcus lesions are, however, unable to use remote reinforcement information to make a correct choice (Kennerley et al. 2006). Our current study further suggests that while the reinforcement protocol is fixed and not dynamically altered in this multiple pair-associate paradigm, the synaptic transmissions in ACC is still important in retrieving and updating the reward-associated spatial information in the schema.

A detailed point of difference from the earlier study (Tse et al. 2011) is the significant increase of Zif268 expression in HPC in the present study. The discrepancy can be explained by reference to three subtle but important differences in procedures-all designed to make the experiences among animals as similar as possible to allow unbiased interpretation of the result. First and foremost, the control group was constituted from animals that had experienced training (current study), rather than being only a caged-control group (Tse et al. 2011). Hence, on the critical day for brain collection (Fig. 2A), animal handling, transportation or exposure to environmental cues should present similar familiarity for and have similar influence on all animals. Second, on the critical day, the control group here was handled at the time when the other two experimental groups had behavior training rather than remaining undisturbed in their home cages. This way, the increase of Zif268 expression in the experimental groups is more likely to reflect the key factor of PAs exposure. Third, all animals in this study had extended training of the original schema and equal experiences of many new PAs, whereas the previous study examined animals that had just completed learning the schema with or without experience of new PAs. With all these factors carefully controlled, we now observed an increase of Zif268 expression in HPC. In fact, this task-related increase in Zif268 expression in HPC is consistent with observations in the watermaze reflecting involvement of HPC in navigational spatial memory (Clark et al. 2005; Martin et al. 2005; Teixeira et al. 2006).

While our data are consistent with both the standard and the complementary learning systems accounts of systems consolidation (McClelland et al. 1995), the very rapid consolidation observed raises the question of whether systems consolidation always requires gradual "reorganization" after learning. Alternatively, network creation could be "experience-expectant" in occurring before new learning that occurs in the future. Our findings on paired-associate learning now consistently point to this possibility (Tse et al. 2007, 2011). IEG activation in both ACC and HPC is consistent with the multiple trace theory (Nadel and Moscovitch 1997); however, the failure to see memory of new
PAs when tested $24 \mathrm{~h}$ later after impairment of ACC neural transmission is challenging to this theory, as hippocampal traces should have been sufficient for readout of memory at such an early stage of systems consolidation. A new schema theory (Wang and Morris 2010) is emerging that reflects the necessity, sufficiency, and engagement of hippocampal-neocortical interactions in the dynamics of schema formation and memory representation (Supplemental Fig. S6).

\section{Acknowledgments}

We thank Patrick Spooner and Jane Tulloch for technical support. This work was supported by grants from the Medical Research Council, UK (R.G.M.M.) and fellowships from the Caledonian Research Foundation and Royal Society of Edinburgh (S.-H.W.).

Author contributions: S.-H.W. and R.G.M.M. conceptualized the study and wrote the manuscript. S.-H.W. designed experiments and performed the procedures, surgery, and data analyses. D.T. performed the early part of behavior training and conducted drug infusions.

\section{References}

Bransford JD. 1979. Human cognition: learning, understanding and remembering. Wadsworth, Belmont, CA.

Clark RE, Broadbent NJ, Squire LR. 2005. Hippocampus and remote spatial memory in rats. Hippocampus 15: 260-272.

Frankland PW, Bontempi B. 2005. The organization of recent and remote memories. Nat Rev Neurosci 6: 119-130.

Kennerley SW, Walton ME, Behrens TE, Buckley MJ, Rushworth MF. 2006 Optimal decision making and the anterior cingulate cortex. Nat Neurosci 9: 940-947.

Martin SJ, de Hoz L, Morris RG. 2005. Retrograde amnesia: Neither partial nor complete hippocampal lesions in rats result in preferential sparing of remote spatial memory, even after reminding. Neuropsychologia 43: 609-624.

Maviel T, Durkin TP, Menzaghi F, Bontempi B. 2004. Sites of neocortical reorganization critical for remote spatial memory. Science 305: 96-99.

McClelland JL, McNaughton BL, O'Reilly RC. 1995. Why there are complementary learning systems in the hippocampus and neocortex: Insights from the successes and failures of connectionist models of learning and memory. Psychol Rev 102: 419-457.

Nadel L, Moscovitch M. 1997. Memory consolidation, retrograde amnesia and the hippocampal complex. Curr Opin Neurobiol 7: 217-227.

Rushworth MF, Behrens TE. 2008. Choice, uncertainty and value in prefrontal and cingulate cortex. Nat Neurosci 11: 389-397.

Squire LR, Alvarez P. 1995. Retrograde amnesia and memory consolidation: A neurobiological perspective. Curr Opin Neurobiol 5: 169-177.

Tang J, Ko S, Ding HK, Qiu CS, Calejesan AA, Zhuo M. 2005. Pavlovian fear memory induced by activation in the anterior cingulate cortex. $\mathrm{Mol}$ Pain 1: 6. doi: 10.1186/1744-8069-1-6.

Teixeira CM, Pomedli SR, Maei HR, Kee N, Frankland PW. 2006. Involvement of the anterior cingulate cortex in the expression of remote spatial memory. J Neurosci 26: 7555-7564.

Tse D, Langston RF, Kakeyama M, Bethus I, Spooner PA, Witter M, Wood ER, Morris RGM. 2007. Schemas and memory consolidation. Science 316: 76-82.

Tse D, Takeuchi T, Kakeyama M, Kajii Y, Okuno H, Tohyama C, Bito H, Morris RG. 2011. Schema-dependent gene activation and memory encoding in neocortex. Science 333: 891-895.

van Kesteren MT, Rijpkema M, Ruiter DJ, Fernandez G. 2010. Retrieval of associative information congruent with prior knowledge is related to increased medial prefrontal activity and connectivity. J Neurosci 30: $15888-15894$.

Wang SH, Morris RG. 2010. Hippocampal-neocortical interactions in memory formation, consolidation, and reconsolidation. Annu Rev Psychol 61: 49-79.

Wang SH, Teixeira CM, Wheeler AL, Frankland PW. 2009. The precision of remote context memories does not require the hippocampus. Nat Neurosci 12: 253-255.

Received March 9, 2012; accepted in revised form May 30, 2012. 


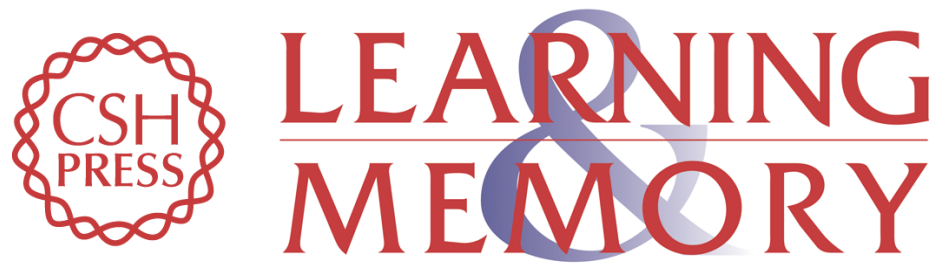

\section{Anterior cingulate cortex in schema assimilation and expression}

Szu-Han Wang, Dorothy Tse and Richard G.M. Morris

Learn. Mem. 2012, 19:

Access the most recent version at doi:10.1101//m.026336.112

Supplemental http://learnmem.cshlp.org/content/suppl/2012/07/13/19.8.315.DC1

Material

References This article cites 16 articles, 5 of which can be accessed free at: http://learnmem.cshlp.org/content/19/8/315.full.html\#ref-list-1

License

Email Alerting Receive free email alerts when new articles cite this article - sign up in the box at the Service top right corner of the article or click here. 